\title{
Involvement of interaction between Fractalkine and CX3CR1 in cytotoxicity of natural killer cells against tumor cells
}

\author{
XIAOJUN ZHANG, HAIMING WEI, HUI WANG and ZHIGANG TIAN \\ Hefei National Laboratory for Physical Sciences at Microscale and School of Life Sciences, \\ University of Science and Technology of China, Hefei 230027, P.R. China
}

Received August 12, 2005; Accepted October 10, 2005

\begin{abstract}
Many chemokine receptors are typically found on natural killer cells, including CX3CR1, the receptor for the chemokine Fractalkine (FKN). This study explored whether interaction between CX3CR1 and FKN is relevant for NK cell functions in cytotoxicity against tumors. FKN expression was examined by polymerase chain reaction and CX3CR1 expression in NK cells was analyzed by flow cytometry. NK cell cytotoxicity was examined by $4-\mathrm{h}{ }^{51} \mathrm{Cr}$-release assay. FKN was expressed in a variety of tumor cell lines such as K562 cells, an NK-sensitive cell line. Approximately $90 \%$ of peripheral blood NK cells and almost all of the NK cell line, NK-92 cells, expressed CX3CR1. Anti-CX3CR1 antibody strongly neutralized the cytotoxicity of NK cells against K562 cells, and pretreatment of NK cells with recombinant soluble FKN improved the cytolytic function on tumor cells. This study demonstrates that an interaction between CX3CR1 on NK cells and FKN on tumor cells is involved in the natural cytotoxicity of NK cells against tumors.
\end{abstract}

\section{Introduction}

Chemokines are a complex family of small regulatory proteins, produced by different cell types, and their receptors are all G protein coupled receptors. The known chemokine system in humans comprises approximately 50 ligands and $20 \mathrm{G}$ proteincoupled receptors (1). The principal targets of chemokines are bone marrow-derived cells. The chemokine system is involved in the recruitment, activation and chemotaxis of leukocytes to the inflammation sites, controlling inflammatory trafficking, which plays a central role in leukocyte physiology. Based on their structural and genetic characteristics, four

Correspondence to: Dr Zhigang Tian, School of Life Sciences, University of Science and Technology of China, 443 Huangshan Road, Hefei City, Anhui 230027, P.R. China

E-mail: tzg@ustc.edu.cn

Abbreviations: PBMC, peripheral blood mononuclear cells; AICD, activation-induced cell death

Key words: chemokine, Fractalkine, CX3CR1, nature killer cells, tumor families of chemokines have been so far identified; c, cxc, cxxc and cxxxc. The receptors via which they function are also divided into four groups, correspondingly (2). Fractalkine is a newly identified chemokine, the only member of the cxxxc family and also known as CX3CL1 $(3,4)$. There are two forms of Fractalkine: the membrane form of Fractalkine, which is anchored to the membrane and may act as an adhesion molecule (5); and the soluble form of Fractalkine, which is released, presumably by proteolysis at a membrane proximal region by TNF- $\alpha$-converting enzyme [TACE (ADAM17) and ADAM10] (6,7). The soluble form of Fractalkine exhibits efficient chemotactic activity for monocytes, NK cells and T cells (3).

Fractalkine is reported to be an important factor during the inflammation process $(3,8)$, in which it complexly interacts with inflammation cytokines (9-12). In some situations, it acts as an inflammation factor and causes cell injury or apoptosis (13) while, in other situations, Fractalkine treatment maintains cell survival and inhibits Fas ligand-induced cell death (14). Fractalkine is also found in amniotic fluid during pregnancy where anti-inflammation factors are needed (15). According to these findings, Fractalkine may be a chemokine with complex functions.

Natural killer (NK) cells are a subgroup of lymphocytes that contribute to protective responses against a variety of infections and cancers (16-20). They are distinguished from $\mathrm{T}$ cells by their participation in innate immunity and early defense. NK cells were originally identified by their ability to spontaneously mediate lysis of certain susceptive tumor cell lines and their large granular lymphocyte morphology (16). CX3CR1 was reported to be expressed on NK cells of PBMCs and may be the media of endothelial cell injury by NK cells with the anchoring form Fractalkine $(5,20)$. NK cells also showed a chemotaxis response to soluble Fractalkine. We also found Fractalkine expressed in many tumor cell lines (21). In this study, we further report that interaction between CX3CR1 and FKN on NK cells and tumor cells is involved in the natural killing process of NK cells against tumors.

\section{Materials and methods}

Abs and reagents. The antibodies against human Fractalkine were purchased from R\&D Systems. The mAb, MF365, was the detective agent in Westen blotting and FACS assay. Rabbit pAb, TP502, purchased from Torrey Pines Biolabs, 
was used to detect human CX3CR1 and to block its biofunction. Secondary antibodies were purchased from BD Biosciences (for FACS) or from SABC (for Western blot). All culture media were purchased from Gibco BRL. Recombinant human sFractalkine was purchased from Peprotech. The kit for reverse transcription was from Invitrogen and the PCR kit was from BBI.

Cells and cell culture. The NK cell line (NK-92) was obtained from ATCC. The tumor cell line (K562) had been maintained in our laboratory for many years. K562 cells were cultured in complete RPMI-1640 medium supplemented with $10 \%$ heat inactivated fetal bovine serum (FBS), $2 \mathrm{mmol} / \mathrm{l} \mathrm{L}$-glutamine, penicillin $(100 \mathrm{U} / \mathrm{ml})$ and streptomycin $(100 \mathrm{U} / \mathrm{ml})$. NK-92 cells were maintained in $\alpha$-minimum essential modified $(\alpha-$ MEM) medium with $12.5 \%$ FBS, $12.5 \%$ horse serum, $100 \mathrm{U} /$ $\mathrm{ml} \mathrm{IL-2,} \mathrm{and} \mathrm{streptomycin} \mathrm{(100} \mathrm{U/ml).}$

Cell-mediated cytotoxicity assay. The lytic capacity of NK cells was examined in a ${ }^{51} \mathrm{Cr}$-release assay. Briefly, target cells (K562) were labeled with ${ }^{51} \mathrm{Cr}$ for $60 \mathrm{~min}$ at $37^{\circ} \mathrm{C}$. Then target cells $\left(10^{4} /\right.$ well) and effector cells were plated onto 96-well plates at the indicated E:T cell ratio and incubated for $4 \mathrm{~h}$ at $37^{\circ} \mathrm{C}$ in a humidified atmosphere containing $5 \%$ $\mathrm{CO}_{2}$. In some experiments, sFractalkine was included in the medium at the indicated concentrations. In experiments using Abs or sFractalkine, NK cells were pretreated with saturating amounts of Abs or sFractalkine for $30 \mathrm{~min}$ at room temperature before the assay.

Reverse transcriptase-polymerase chain reaction. Briefly, total RNA was isolated from NK cells or K562 cells by using TRIzol (Gibco BRL). Five micrograms of RNA was reversely transcribed by random primer and incubated with $200 \mathrm{U}$ M-MLV for $1 \mathrm{~h}$ at $37^{\circ} \mathrm{C}$. Ten micrograms of single-stranded cDNA was then subjected to 30 cycles of PCR. Samples were denatured for $3 \mathrm{~min}$ at $95^{\circ} \mathrm{C}$ and, after addition of 2-U polymerase to a total volume of $100 \mu 1$ reaction medium, the mixture was subjected to a 30-cycle PCR amplification program, each containing $30-\mathrm{sec}$ denaturing at $94^{\circ} \mathrm{C}, 30-\mathrm{sec}$ annealing at different temperatures, and 1-min extension at $72^{\circ} \mathrm{C}$, with a 7 -min extension after the last cycle. The primer sequences were: human Fractalkine (306 bp), forward 5'-AG GAGAATGCTCCGTCTGAA-3' and reverse 5'-AGAAGAG GAGGCCAAGGAAG-3'; human CX3CR1, forward 5'-TGG TAAAGTCTGAGCAGGACAGG-3' and reverse 5'-GTTGT TCATGGAGTTGGCGG-3'; and human B-actin, forward 5'GACCTGACTGACTACCTCATGAAGAT-3' and reverse 5'-GTCACACTTCATGATGGAGTTGAAGG-3'.

Flow cytometry analysis. To examine the surface expression of CX3CR1, NK-92 cells were first stained with the primary antibody, anti-CX3CR1 pAb, and then with the PE-conjugated secondary antibody. All cells were analyzed using a FACSCalibur flow cytometer (BD Biosciences) and data were analyzed by WinMDI (free software for FACS analysis).

Statistical analysis. Student's t-tests for cytotoxicity were performed to determine the statistical difference with $\mathrm{p}<0.05$ or $\mathrm{p}<0.01$ being considered significant or highly significant,

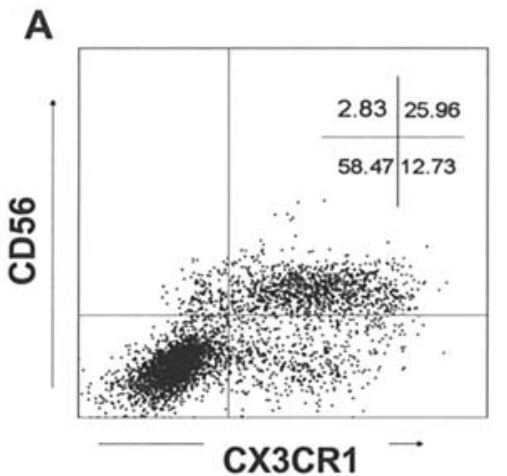

B

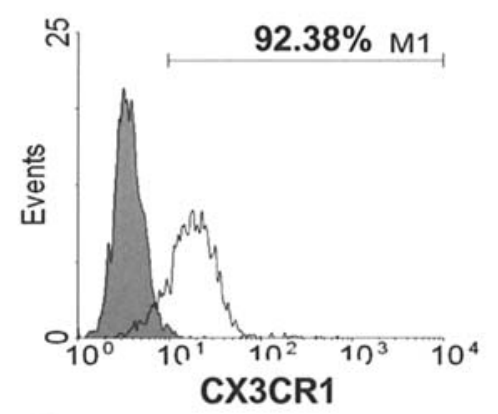

C

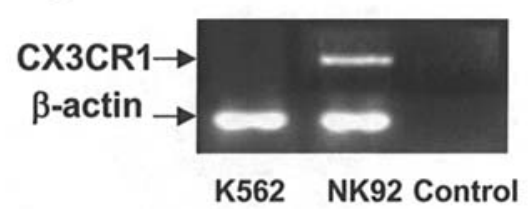

Figure 1. Expression of CX3CR1 on NK cells. (A) The PBMC was isolated and then stained with anti-CD56 and anti-CX3CR1 Abs. The lymphocytes were gated and the $\mathrm{CD} 56^{+} \mathrm{CX} 3 \mathrm{CR} 1^{+}$cells were assayed by flow cytometry. 90.18\% of NK cells were CX3CR1 positive [25.96/(25.96+2.83) x 100\%]. (B) NK-92 cells were stained with anti-CX3CR1 pAb and then assayed. $92.38 \%$ of NK-92 cells were positive. (C) mRNA levels of NK-92 cells by RT-PCR analysis. Total cell RNA was abstracted from NK-92 cells or K562 cells (control cells), then reversely transcribed (RT) into cDNA and finally amplified by PCR method. In order to exclude the contamination, we also had a negative control group which did not practice RT procedure.

respectively. All experiments were performed at least three times with a representative experiment being shown.

\section{Results}

Expression of CX3CRI on NK cells and Fractalkine on tumor cells. To confirm the expression of CX3CR1 on NK cells, mononuclear cells were isolated from human peripheral blood and the expression was confirmed by flow cytometry, using the antibody against CD56 and antibody against CX3CR1. As shown in Fig. 1A, 90.18\% of NK cells were CX3CR1 positive [25.96/(25.96+2.83) x 100\%], indicating that most human blood NK cells expressed surface CX3CR1 proteins. Meanwhile, we also examined the surface expression of CX3CR1 on human NK cell line, NK-92 cells. As shown in Fig. 1B, NK-92 cells expressed a high level of membrane CX3CR1 molecules. These results were further confirmed by RT-PCR analysis and the PCR product with the predicted length was obtained from the total RNA of NK-92 cells (Fig. 1C). 


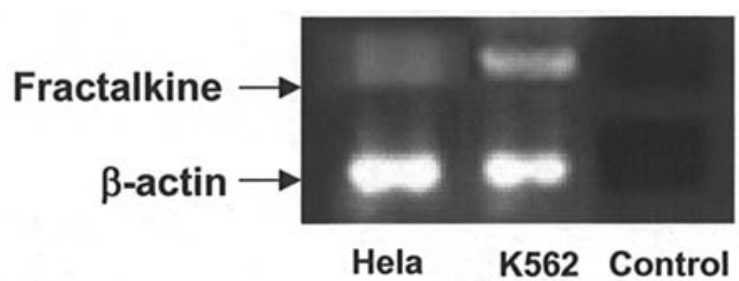

Figure 2. Expression of Fractalkine mRNA in tumor cells. Total cell RNA was abstracted from HeLa cells or K562 cells, then reversely transcribed (RT) into cDNA, and finally amplified by PCR method. In order to exclude the contamination, we also had a negative control group which did not practice RT procedure.

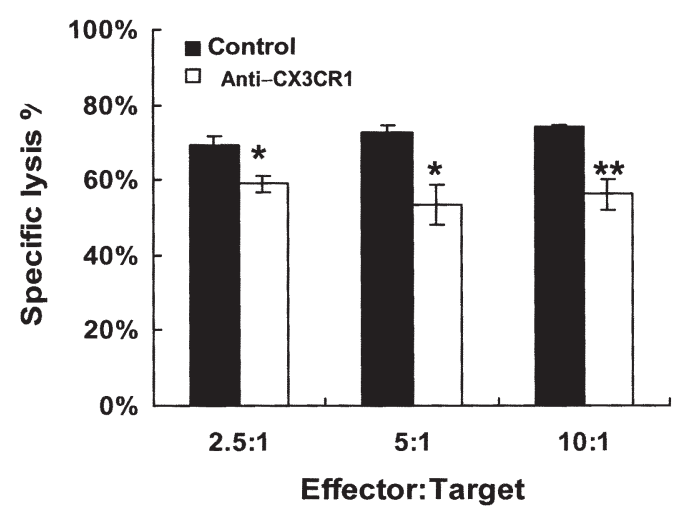

Figure 3. Anti-CX3CR1 antibody neutralizes the cytotoxicity of NK-92 cells against K562 tumor cells. The natural cytotoxic activity of NK-92 cells against K562 cells was evaluated using $4-\mathrm{h}{ }^{51} \mathrm{Cr}$-release assays at each effector:target ratio in the presence or absence of anti-CX3CR1 antibody. Values represent the means from at least 3 independent experiments.

In order to verify if $\mathrm{K} 562$, the killing sensitive target cells of NK cells, express Fractalkine, we performed RT-PCR assay. The predicted length of RT-PCR product was obtained from total RNA of K562 (Fig. 2). The results were also confirmed by Western blotting (data not shown).

\section{Effects of neutralization and activation of CX3CR1-Fractalkine} interaction on cytotoxicity of $\mathrm{NK}$ cells against tumor cells. To explore the possible involvement of CX3CR1-Fractalkine interaction in the natural cytotoxicity against tumors, a neutralizing antibody was used in 4-h cytotoxicity assay system with NK-92 cells against K562 tumor cells. As shown in Fig. 3, the neutralizing antibody partly blocked the cytotoxicity of NK-92 cells against K562 tumor cells. The inhibitory effects of the neutralizing antibody on NK-92K562 interaction were greater at a higher effector:target ratio, indicating that $\mathrm{CX} 3 \mathrm{CR} 1-$ Fractalkine interaction was possibly involved in effector/target interaction (Fig. 3).

In order to directly correlate the CX3CR1-Fractalkine interaction to the natural cytotoxicity of freshly isolated PBMC against K562 tumor cells, we then stimulated the PBMC with recombinant soluble Fractalkine in vitro. As shown in Fig. 4, the natural cytotoxic activity of PBMC pretreated with recombinant soluble Fractalkine $(40 \mathrm{ng} / \mathrm{ml})$ against K562 cells was significantly enhanced at each effector:target ratio in $4-\mathrm{h}{ }^{51} \mathrm{Cr}$-release assays, indicating that Fractalkine may activate the NK cells in the killing process.

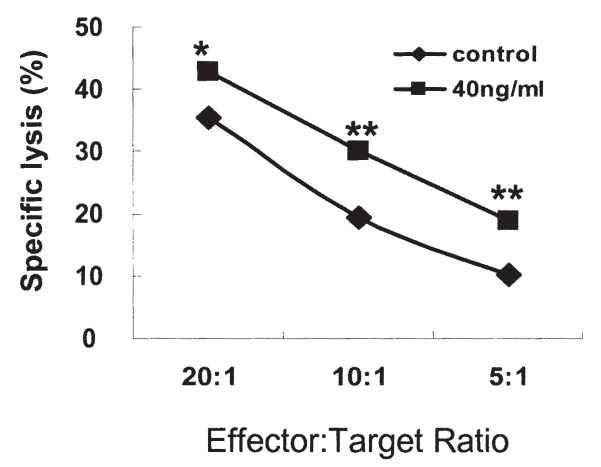

Figure 4. Recombinant soluble Fractalkine (rsFNK) improves the cytotoxicity of PBMC against K562 tumor cells. The natural cytotoxic activity of PBMC pretreated with or without recombinant soluble Fractalkine (40 ng/ml) against K562 cells was evaluated using 4-h ${ }^{51} \mathrm{Cr}$-release assays at each effector:target ratio. Values represent the means from at least 3 independent experiments.

\section{Discussion}

The importance of FKN/CX3CR1 interaction was explored in endothelium. Leukocyte trafficking at the endothelium requires both cellular adhesion molecules and chemotactic factors. FKN induced both adhesion and migration of leukocytes. Imai et al (5) firstly identified a seven-transmembrane high-affinity receptor for FKN, then termed CX3CR1, and showed that it mediated both the adhesive and migratory functions of FKN to endothelia cells (ECs). They also observed that NK cells predominantly expressed CX3CR1 and responded to FKN in both migration and adhesion (5). It was reported that CX3CR1 was preferentially expressed in Th1 compared with Th2 cells. Th1 but not Th2 cells responded to FKN. FKN was expressed in ECs in psoriasis, a Th1-dominated skin disorder, but not in Th2-driven atopic dermatitis (5). Immobilized FKN by transfecting ENs markedly induced IFN- $\gamma$ production by NK cells in a dose-dependent manner. Pretreatment of NK cells with the phosphatidylinositol 3kinase (PI 3-K) inhibitor completely inhibited the production of IFN- $\gamma$ induced by Fractalkine (11). Transfection of FKN cDNA into ECV304 cells or HUVECs resulted in increased adhesion of NK cells and susceptibility to NK cell-mediated cytolysis compared with control transfection. Moreover, both enhanced adhesion and susceptibility of Fractalkinetransfected cells were markedly suppressed by anti-CX3CR1 $\mathrm{Ab}$ (21). These findings demonstrate that FKN/CX3CR 1 interaction plays an important role in the recruitment and adhesion of leukocytes including NK cells in inflammation.

More attention has been paid to the application potential of FKN in tumor immunotherapy. Several murine tumor models have been explored. It was found that radiolabelled YAC-1 target cells intravenously injected into C57BL/6 mice rapidly localized to the lungs and were cleared by NK cells. In mice pretreated with blocking anti-FKN or anti-CX3CR1 Ab, target cell clearance decreased by 4- to 5-fold, which correlated with decreased NK cell binding to activated endothelium (22). When murine lymphoma cell lines (EL4 and its derivative EG7) stably transfected with human-CX3CL1 were injected s.c. into C57BL/6 mice, the tumor growth was severely impaired. This antitumor effect of CX3CL1 was also found 
in $\mathrm{T}$ and $\mathrm{B}$ cell-deficient $\mathrm{Rag} 1^{-/-}$mice but vanished in natural killer (NK) cell-deficient beige mice and in $\mathrm{CX} 3 \mathrm{CR}^{-/-}$mice, suggesting the involvement of CX3CR1-expressing NK cells (5). Guo et al $(27,28)$ further found that FNK in a soluble form can chemoattract T cells and DC and membrane-bound FKN can adhere T cells and DC. NK activity increased in mice inoculated with 3LL-FKN, and in vivo depletion of NK cells resulted in the decreased tumor growth inhibition of 3LL-FK. Incubation of NK cells with 3LL-FKN could increase the cytotoxicity of NK cells against YAC-1 cells and even against NK-resistant parental 3LL cells (12,23). Recently, Xin et al (29) observed that intratumoral injection of adenoviral vector expressing mouse FKN (AdFKN) into C26 and B16F10 tumors resulted in a marked reduction of tumor growth. Histological examination of tumor tissue revealed abundant infiltration of NK cells, dendritic cells, and CD8 (+) T lymphocytes. Antitumor effects by AdFKN were completely abrogated in both NK cell-depleted mice and CD8 (-/-) mice, and partially blocked in CD4 (-/-) mice. These data indicated that Fractalkine mediates antitumor effects by both NK celldependent and $\mathrm{T}$ cell-dependent mechanisms (24). Though almost all experiments were conducted on mice, Ohta et al (30) reported the first clinical results. Colorectal cancer (CRC) cases with a strong FKN expression showed a significantly better prognosis than those with a weak expression. Fractalkine expressed in the tumor appears to recruit cytotoxic $\mathrm{T}$ cells and NK cells to the tumor site and these cytotoxic cells result in a better prognosis mediated by tumor cell cytotoxicity (25). These human results from clinical data analysis greatly supported those obtained from mice. Our in vitro human study further confirms the results in mice and humans, and demonstrates a direct interaction between FKN in human tumor cells and CX3CR1 in human NK cells.

\section{Acknowledgements}

This work was supported by the Natural Science Foundation of China (\#30125038, \#30230340, \#30371302, \#30371308), the Key Basic Science Program of the Ministry of Science and Technology of China (\#2001CB510009, \#2003CB515501) and the Foundation of the Chinese Academy of Science (\#KSCX2-2-08).

\section{References}

1. Gerard C and Rollins BJ: Chemokines and disease. Nat Immunol 2: 108-115, 2001.

2. Rossi D and Zlotnik A: The biology of chemokines and their receptors. Annu Rev Immunol 18: 217-242, 2000.

3. Bazan JF, Bacon KB, Hardiman G, et al: A new class of membrane-bound chemokine with a CX3C motif. Nature 385: 640-644, 1997.

4. Pan Y, Lloyd C, Zhou H, et al: Neurotactin, a membraneanchored chemokine upregulated in brain inflammation. Nature 387: 611-617, 1997.

5. Imai T, Hieshima K, Haskell C, et al: Identification and molecular characterization of fractalkine receptor CX3CR1, which mediates both leukocyte migration and adhesion. Cell 91: 521-530, 1997.

6. Garton KJ, Gough PJ, Blobel CP, et al: Tumor necrosis factoralpha-converting enzyme (ADAM17) mediates the cleavage and shedding of fractalkine (CX3CL1). J Biol Chem 276: 37993-38001, 2001 .
7. Tsou CL, Haskell CA and Charo IF: Tumor necrosis factoralpha-converting enzyme mediates the inducible cleavage of fractalkine. J Biol Chem 276: 44622-44626, 2001.

8. Chapman GA, Moores K, Harrison D, Campbell CA, Stewart BR and Strijbos PJ: Fractalkine cleavage from neuronal membranes represents an acute event in the inflammatory response to excytotoxic brain damage. J Neurosci 20: RC87, 2000.

9. Soriano SG, Amaravadi LS, Wang YF, et al: Mice deficient in fractalkine are less susceptible to cerebral ischemia-reperfusion injury. J Neuroimmunol 125: 59-65, 2002.

10. Ludwig A, Berkhout T, Moores K, Groot P and Chapman G: Fractalkine is expressed by smooth muscle cells in response to IFN-gamma and TNF-alpha and is modulated by metalloproteinase activity. J Immunol 168: 604-612, 2002.

11. Matsumiya $\mathrm{T}$, Imaizumi $\mathrm{T}$, Fujimoto $\mathrm{K}$, et al: Soluble interleukin-6 receptor alpha inhibits the cytokine-induced fractalkine/CX3CL1 expression in human vascular endothelial cells in culture. Exp Cell Res 269: 35-41, 2001.

12. Yoneda O, Imai T, Nishimura M, et al: Membrane-bound form of fractalkine induces IFN-gamma production by NK cells. Eur J Immunol 33: 53-58, 2003.

13. Zujovic V, Benavides J, Vige X, Carter C and Taupin V: Fractalkine modulates TNF-alpha secretion and neurotoxicity induced by microglial activation. Glia 29: 305-315, 2000.

14. Umehara $\mathrm{H}$ and Imai $\mathrm{T}$ : Role of fractalkine in leukocyte adhesion and migration and in vascular injury. Drug News Perspect 14: 460-464, 2001.

15. Boehme SA, Lio FM, Iejewski-Lenoir D, Bacon KB and Conlon PJ: The chemokine fractalkine inhibits Fas-mediated cell death of brain microglia. J Immunol 165: 397-403, 2000.

16. Shimoya K, Zhang Q, Tenma K, et al: Fractalkine (FRK) levels in amniotic fluid and its production during pregnancy. Mol Hum Reprod 9: 97-101, 2003.

17. Trinchieri G: Biology of natural killer cells. Adv Immunol 47: 187-376, 1989.

18. Biron CA: Activation and function of natural killer cell responses during viral infections. Curr Opin Immunol 9: 24-34, 1997.

19. Unanue ER: Inter-relationship among macrophages, natural killer cells and neutrophils in early stages of Listeria resistance. Curr Opin Immunol 9: 35-43, 1997.

20. Scharton-Kersten TM and Sher A: Role of natural killer cells in innate resistance to protozoan infections. Curr Opin Immunol 9: 44-51, 1997.

21. Scott P and Trinchieri G: The role of natural killer cells in hostparasite interactions. Curr Opin Immunol 7: 34-40, 1995

22. Efsen E, Grappone C, De Franco RM, et al: Up-regulated expression of fractalkine and its receptor CX3CR1 during liver injury in humans. J Hepatol 37: 39-47, 2002.

23. Fraticelli P, Sironi M, Bianchi G, et al: Fractalkine (CX3CL1) as an amplification circuit of polarized Th1 responses. J Clin Invest 107: 1173-1181, 2001.

24. Yoneda O, Imai T, Goda S, et al: Fractalkine-mediated endothelial cell injury by NK cells. J Immunol 164: 4055-4062, 2000.

25. Robinson LA, Nataraj C, Thomas DW, et al: The chemokine CX3CL1 regulates NK cell activity in vivo. Cell Immunol 225: 122-130, 2003.

26. Lavergne E, Combadiere B, Bonduelle O, et al: Fractalkine mediates natural killer-dependent antitumor responses in vivo. Cancer Res 63: 7468-7474, 2003.

27. Guo J, Chen T, Wang B, et al: Chemoattraction, adhesion and activation of natural killer cells are involved in the antitumor immune response induced by fractalkine/CX3CL1. Immunol Lett 89: 1-7, 2003

28. Guo J, Zhang M, Wang B, et al: Fractalkine transgene induces T-cell-dependent antitumor immunity through chemoattraction and activation of dendritic cells. Int J Cancer 103: 212-220, 2003.

29. Xin H, Kikuchi T, Andarini S, et al: Antitumor immune response by CX3CL1 fractalkine gene transfer depends on both NK and T cells. Eur J Immunol 35: 1371-1380, 2005.

30. Ohta M, Tanaka F, Yamaguchi H, Sadanaga N, Inoue H and Mori M: The high expression of Fractalkine results in a better prognosis for colorectal cancer patients. Int J Oncol 26: 41-47, 2005 . 